Cahiers de recherches médiévales

\title{
Cuisine et médecine au Moyen Âge
}

Alliées ou ennemies?

\section{Bruno Laurioux}

\section{CpenEdition}

Journals

Édition électronique

URL : https://journals.openedition.org/crm/862

DOI : $10.4000 / \mathrm{crm} .862$

ISSN : 1955-2424

Éditeur

Honoré Champion

Édition imprimée

Date de publication : 30 mars 2006

Pagination : 223-238

ISSN : $1272-9752$

Référence électronique

Bruno Laurioux, «Cuisine et médecine au Moyen Âge », Cahiers de recherches médiévales [En ligne], 13 spécial | 2006, mis en ligne le 03 avril 2009, consulté le 15 décembre 2022. URL : http://

journals.openedition.org/crm/862 ; DOI : https://doi.org/10.4000/crm.862 


\section{酷M}

\section{Cuisine et médecine au Moyen Âge : alliées ou ennemies?}

Concilier santé et bon goût est un idéal largement répandu dans la société actuelle, sans pouvoir être si souvent atteint tant les exigences gastronomiques et médicales semblent contradictoires. À première vue, cette contradiction n'apparaît pas au Moyen Âge, ce qui a fait dire à certains historiens de l'alimentation - et non des moindres (Jean-Louis Flandrin'1, Ria Jansen-Sieben ${ }^{2}$, Terence Scully ${ }^{3}$ et bien d'autres) - que, au fond, la cuisine médiévale n'était qu'une diététique en action.

Lorsqu'on y regarde de près, le tableau se révèle plus complexe. Entre cuisine et médecine, se découvrent ainsi beaucoup de diversités (d'approches, de prémisses), de divergences (dans les textes, pour la combinaison des ingrédients) et même des désaccords. On connaît ce passage du commentaire au Canon d'Avicenne où Jacques Despars se plaint de ce que le régime prescrit par les Anciens après une chute ne soit plus respecté de son temps : "Soit parce que, explique-t-il, les hommes de notre temps, adonnés à la gloutonnerie, ne sont plus habitués à subir d'importantes abstinences. Soit parce que leurs serviteurs, et surtout leurs femmes, ne croient jamais avoir assez empli d'aliments le ventre des malades et s'élancent de leur langue [médisent], en cachette ou ouvertement, contre ceux qui ont imposé ces diètes strictes... ${ }^{4}$. Examiner les différences et les points communs entre approches médicales et culinaires, et surtout les rencontres - hostiles ou amicales - ainsi que les points de passage qui relièrent au Moyen Âge les deux domaines : tel est le but de ce dossier 5 .

Le regard que l'on a voulu privilégier est, pour une fois, celui des historiens de la médecine. Spécialiste reconnue de la diététique médiévale dont elle est sans doute à l'heure actuelle la meilleure connaisseuse, Marilyn Nicoud décrypte tout en

\footnotetext{
${ }^{1}$ J.-L. Flandrin, «Diététique et gastronomie, XIV ${ }^{\mathrm{e}}-\mathrm{XVIII}{ }^{\mathrm{e}}$ siècles », Voeding en Geneeskunde. Alimentation et médecine, Actes du colloque de Bruxelles, 12.10.1990, éd. R. Jansen-Sieben et F. Daelemans, Bruxelles (Archives et bibliothèques de Belgique $\mathrm{n}^{\circ}$ spécial 41), 1993, p. 177-92.

${ }^{2}$ R. Jansen-Sieben, «From food therapy to cookery-book», Medieval Dutch Literature in its European Context, éd. E. Kooper, Cambridge, 1994, p. 261-279; article publié antérieurement en néerlandais dans Voeding en Geneeskunde, Op. cit., p. 125-51 et postérieurement en allemand dans les Würzburger medizinhistorische Mitteilungen, 16, 1997, p. 191-202

${ }^{3}$ T. Scully, "Mixing it up in Medieval Kitchen », dans Medieval Food and Drink, éd. M.-J. Arn, Acta (The Center for Medieval and Early Renaissance Studies, Binghamton University, State University of New York), 21, 1995, p. 1-26 ; Id., The Art of Cookery in the Middle Ages, Woodbridge-Rochester, 1995.

${ }^{4}$ Cité par D. Jacquart, La Médecine médiévale dans le cadre parisien, XIV $-X V^{e}$ siècle, Paris, 1998, p. 433.

5 Les articles qui suivent sont issus de communications prononcées aux premières «Rencontres sur la cuisine médiévale» de Châteaudun (23 octobre 2003). C'est un plaisir pour moi de remercier M. Gilles de Langsdorff, administrateur du château de Châteaudun qui a généreusement accueilli cette manifestation et $M$. Gabriel Lefrancq qui en a grandement facilité la réalisation.
}

Cahiers de Recherches Médiévales, 13spé, 2006 
finesse les rapports complexes que ce savoir - objet d'une curiosité intense à la fin du Moyen Âge, aussi bien dans le grand public que dans les milieux universitaires entretenait alors avec les pratiques alimentaires. La frontière reste souvent floue entre médecine et cuisine et les mêmes produits, de médicaments peuvent se transformer en aliments : c'est le cas pour un grand nombre de plantes, nous rappelle Mireille Ausécache, qui a mené à bien une importante thèse sur le médecin Gilles de Corbeil. Il fallait toute la rigueur et l'exigence de Danielle Jacquart - qui a d'ailleurs formé ces deux chercheuses - pour nous faire comprendre la théorie de la nutrition sous-tendant la conception que les médecins se faisaient de la cuisine : le cheminement bien réglé qui, de la mastication à l'assimilation, transforme l'aliment en substance vitale n'avait jamais été exposé avec tant de clarté.

Mais peut-être n'est-il pas inutile de rappeler au préalable les certitudes - fort peu nombreuses -, les remises en cause et les débats qu'a bâtis, légués ou connus l'histoire de l'alimentation dans les dernières décennies sur l'épineuse question des rapports entre cuisine et médecine. Pour faire court, tout le monde s'est accordé à en reconnaître l'importance sans que personne consente à la traiter dans tous ses aspects et ses prolongements.

\section{La terminologie : qu'entend-on exactement par cuisine et médecine ?}

La cuisine peut être entendue comme l'ensemble des techniques visant à rendre les aliments consommables - et j'ajouterais : agréables au goût. Elle n'est donc qu'un des éléments de l'alimentation. Pour le Moyen Âge, en-dehors des rares informations à tirer des fouilles archéologiques (traces de découpe et de carbonisation) et des inventaires notariés ${ }^{6}$, on la connaît surtout par les livres de cuisine, qui apparaissent à partir de la fin du $\mathrm{XIII}^{\mathrm{e}}$ siècle et se multiplient jusqu'à la fin du $\mathrm{XV}^{\mathrm{e}}$ siècle, sous forme d'abord manuscrite puis imprimée 7 .

La médecine considérée ici est la médecine savante, la seule à avoir laissé des textes, même s'il n'est pas exclu que des conceptions médicales «empiriques » plus ou moins teintées de médecine savante aient pu interférer avec les pratiques culinaires. Parmi les textes médicaux, il ne faut pas seulement considérer les œuvres proprement diététiques - «régimes de santé » généraux ${ }^{8}$ ou traités spécialisés sur les aliments - mais aussi les régimes individuels ou collectifs composés pour combattre telle ou telle maladie (notamment la peste ${ }^{9}$ ) et ces ordonnances développées que sont

\footnotetext{
${ }^{6}$ Cf. B. Laurioux, Une Histoire culinaire du Moyen Âge, Paris, 2005 (Sciences, techniques et civilisations du Moyen Âge à l'aube des Lumières, 8), p. 9-34.

${ }^{7}$ B. Laurioux, Les Livres de cuisine médiévaux, Turnhout, 1997 (Typologie des sources du Moyen Âge occidental, 77).

${ }^{8}$ P. Gil Sotres, "Les régimes de santé », Histoire de la pensée médicale en Occident, dir. M. D. Grmek, t. I, Antiquité et Moyen Age, Paris, 1995, p. 257-81; D. Jacquart-M. Nicoud, "Les régimes de santé au XIII ${ }^{\mathrm{e}}$ siècle ", Comprendre le XIII siècle. Études offertes à MarieThérèse Lorcin, dir. P. Guichard-D. Alexandre-Bidon dir., Lyon, 1995, p. 201-214; M. Weiss-Adamson, Medieval Dietetics. Food and Drinks in Regimen Sanitatis Literature from 800 to 1400, Francfort, 1995.

${ }^{9}$ Cf. les nombreux textes rassemblés par Karl Sudhoff, dans les 20 fascicules consacrés aux Pestschriften aus den ersten 150 Jahren nach der Epidemie des "schwarzen Todes" et parus dans 1'Archiv für Geschichte der Medizin du n 4 (1910-1911) au nº 17 (1925).
} 
les consilia ${ }^{10}$. Quelle que soit leur forme, ces textes partagent une certaine vision de la nutrition qui donne à la cuisine un rôle fondamental : celui, par les opérations de cuisson et les mélanges qu'elle opère, de contribuer à la conservation de ce fragile équilibre qu'est la santé et aussi de combattre les maladies; la cuisine est ainsi à même de prolonger, de corriger ou d'accélérer le processus de digestion, conçu luimême comme une coction.

La différence entre cuisine et médecine est donc en premier lieu d'ordre documentaire. D'un côté, un nombre somme toute assez réduit de recueils de recettes (environ 150 pour deux siècles et toute l'Europe) qui restent de surcroît peu explicites (du fait des circonstances de leur composition et du public auquel ils s'adressent) et lacunaires - ils ne concernent que les élites et n'intègrent pas toutes les préparations connues de celles-ci. Du côté de la médecine, la masse de textes est impressionnante : on a par exemple repéré 1012 manuscrits médicaux en anglais pour les trois derniers siècles du Moyen Âge ${ }^{11}$, soit un rapport entre manuscrits culinaires et manuscrits médicaux de 1 à 23. Et encore ne tient-on pas compte des 4777 manuscrits médicaux en latin conservés dans les bibliothèques britanniques : le rapport serait alors de 1 à 111 ! Si l'on se limite à la diététique, Marilyn Nicoud a recensé près de 500 manuscrits comportant un texte diététique occidental, c'est-à-dire non traduit de l'arabe ou du grec ${ }^{12}$. Si l'on intégrait à ce décompte des traductions aussi répandues que le Tacuinum Sanitatis ${ }^{13}$, les Diètes d'Isaac ${ }^{14}$ ou le Des facultés des aliments de Galien ${ }^{15}$, on arriverait sans doute à quelques centaines supplémentaires. Même si cette masse documentaire n'est pas encore très bien cernée, elle en impose. Au rebours du livre de cuisine, le texte médical offre en outre l'avantage de la rigueur et de l'explicitation; loin des recettes évasives, les prescriptions médicales sont proportionnées et individualisées.

On s'explique assez bien que la balance ait majoritairement penché du côté de la médecine, dont aux yeux de beaucoup la cuisine n'était au fond qu'un sousproduit. Cette thèse, que je qualifierai volontiers de croyance, peut se critiquer aux

\footnotetext{
${ }^{10}$ J. Agrimi-C. Crisciani, Les «Consilia » médicaux, Turnhout, 1994 (Typologie des sources du Moyen Âge occidental, 69).

${ }^{11}$ R. H. Robbins, « Medical manuscripts in Middle English », Speculum, 45, 1970, p. 393, note 2 (rapportant des travaux de Dorothea Waley Singer).

${ }^{12} \mathrm{M}$. Nicoud, Aux origines d'une médecine préventive. Les traités de diététique en Italie et en France (XIII ${ }^{e}-X V^{e}$ siècle), thèse ÉPHÉ IV ${ }^{\mathrm{e}}$ section, Paris, 3 vol., 1998.

${ }^{13}$ Il n'existe pour l'heure aucune étude d'ensemble sur les manuscrits du Tacuinum Sanitatis. Les renseignements que j'ai pu glaner çà et là laissent penser que la version latine longue est conservée dans près d'une vingtaine de manuscrits, la version latine courte et illustrée dans à peine une dizaine de témoins et la version italienne dans quatre codex.

${ }^{14}$ M. Nicoud, Aux origines d'une médecine préventive, op. cit., t. 3, p. 759-69, a décompté 60 manuscrits plus ou moins complets des Diètes universelles et / ou particulières, ainsi que de leurs commentaires. C'est un minimum : cf. par exemple le manuscrit en mains privées reproduit sur le site du Schoenberg Center for Electronic Text and Image, University of Pennsylvania (http://dewey.library.upenn.edu/sceti/ljs024).

${ }^{15}$ H. Diels, Die Handschriften der antike Ärzte, I, Berlin, 1905 (Abhandlungen der Kgl. Preussischen Akad. der Wissenschaften), p. 76-7, recense 37 manuscrits de ce traité auxquels Durling (R. J. Durling, «Corrigenda and Addenda to Diels' Galenica», Traditio, 23, 1967, p. 461-76 et 37, 1981, p. 373-81) ajoute deux témoins - mais en a également ôté deux.
} 
trois niveaux de rencontre entre cuisine et médecine : celui des individus, celui des pratiques, enfin celui des textes.

\section{Le médecin et le cuisinier}

Le médecin de cour a effectivement pour mission de surveiller le travail des cuisines, comme le précisent certaines ordonnances de l'hôtel. Au XIV ${ }^{\mathrm{e}}$ siècle, le dauphin de Viennois Humbert II enjoint à son médecin «qu'il n'oublie pas d'avoir chaque jour une discussion (collationem) avec le maitre de notre hôtel et le maître de la cuisine sur la préparation des mets $»^{16}$. Le terme collatio n'a pas été choisi au hasard : il s'applique notamment à la discussion que les médecins ont entre eux et / ou avec d'autres spécialistes de santé (chirurgiens, sage-femmes) sur un cas particulièrement difficile et qui passe par un entretien avec le malade - une sorte de pendant pratique de la disputatio ${ }^{17}$. C'est ce genre de circonstance qu'a vraisemblablement en tête le rédacteur d'une ordonnance établie pour l'hôtel du roi d'Angleterre Édouard IV : il précise en effet que non seulement le docteur en médecine est chargé de répondre aux questions du roi sur la nature et l'effet des nourritures mais qu' «il doit parler» notamment "avec le maître-queux» (maister cooke) "pour décider par conseil quel mets ou quelle boisson est la mieux appropriée au roi $»^{18}$. Dans les grandes cours, il était donc possible d'expérimenter une cuisine aussi individualisée que le voulait la diététique. Mais sans doute les médecins de cour le faisaient-ils avec une grande souplesse, en cherchant à trouver un équilibre entre les nécessités médicales et les exigences du goût.

Plus délicate à prouver est la culture médicale du cuisinier. Certes, des exemples indubitables s'en trouvent dans la littérature, mais ils y sont présentés comme un idéal: Konrad de Megenberg, dans son Yconomica, affirme qu'un bon cuisinier doit connaître la diététique des saisons, les qualités thérapeutiques des épices et l'ordre des mets requis par l'art médical, toutes informations qu'il ira chercher auprès d'un médecin ${ }^{19}$. Maître Chiquart est en revanche un praticien bien réel, dont la

${ }^{16}$ [Physicus] nec obmittat die qualibet collationem habere cum magistris nostri hospitii et coquinae de cibariis praeparandis, quae nostrae personae magis conferant et utilia reputentur, et frequenter ad oculum videat in horis prandendi et coenandi statum nostrum (cité par Sabine Krüger dans son édition de l'Yconomica de Konrad von Megenberg, Konrad von Megenberg Werke, vol. 5, Ökonomik (Buch I), Stuttgart 1973 [MGH, 500-1500, Staatschriften des Späteren Mittelalters, III, 5, 1], p. 193, note 1).

${ }^{17}$ D. Jacquart, La Médecine médiévale dans le cadre parisien, op. cit., p. 88-92.

${ }^{18}$ «Doctoure of physyque, stondith muche in the King's presence at his meles, councellyng or answering to the King's grace whiche dyet is best according, and to tell the nature and operation of all the metes. And muche he should talke with the steward, chamberlayn, assewer, and the maister cooke, to devyse by counsayle what metes or drinkes is best accordinge with the Kinge » (Liber Niger Domus Regis Edw. IV [1471-1472], éd. dans A Collection of Ordinances and Regulations for the Government of the Royal Household..., Londres, 1790 ; cf. A. R. Myers, The Household of Edward IV. The Black Book and the Ordinance of 1478, Manchester, 1959).

${ }^{19}$ Boni coci symee sunt medicorum, quoniam de cibis sic aut sic condiendis cum aromatibus hiis aut aliis elixando vel assando cocorum est noscere; quia saltem in communibus cibis et medici est propter quod assignare. C'est toute la notice qu'il faudrait ici citer (Konrad von 
personnalité et l'œuvre ont été mises en avant ces dernières années par Terence Scully ${ }^{20}$. Ce cuisinier du premier duc de Savoie, Amédée VIII, sur l'ordre duquel il a entrepris d'écrire le Fait de cuisine, semble a priori avoir une certaine connaissance littéraire et médicale : le manuscrit qui transmet son œuvre comporte ainsi un passage manifestement tiré du Regimen Sanitatis Salernitanum, un poème synthétisant les notions fondamentales d'hygiène ${ }^{21}$. Mais ce manuscrit n'est précisément pas l'original ${ }^{22}$ et les passages en question apparaissent plutôt comme des ajouts du copiste - probablement un lettré, quant à luii ${ }^{23}$.

\section{Principes diététiques et pratique culinaire}

Une première voie d'approche a consisté à chercher dans les recettes que nous fournissent les livres de cuisine une cohérence avec les théories diététiques: c'est la voie qu'a notamment pratiquée Rashmi Patni ${ }^{24}$ et dans une moindre mesure Terence Scully. Elle comporte de multiples dangers, qui n'ont pas toujours été évités.

Au nom du principe - on ne peut plus discutable - selon lequel la médecine médiévale aurait été intangible durant plus d'un millénaire, on n'a pas hésité à comparer des textes médicaux antiques (et qui plus est dans leur traduction française de la Renaissance !) avec des recettes des $\mathrm{XIV}^{\mathrm{e}}$ et $\mathrm{XV}^{\mathrm{e}}$ siècles. Pour la cohérence du propos, il faut limiter la comparaison à des textes contemporains - et, pour les textes médicaux, à des textes susceptibles d'être connus au Moyen Âge, ce qui exclue les ouvrages grecs non traduits avant la Renaissance.

Deuxième écueil, les catégorisations culinaires et médicales ne se recouvrent pas entièrement. Rashmi Patni analyse, par exemple, les recettes culinaires en fonction de la grille des saveurs médicales, qui peuvent aller jusqu'au nombre de neuf (l'acerbe, l'austère, l'acide, l'insipide, la grasse et la douce, la salée, l'amère et l'âcre ou acerbe $)^{25}$. Or, elles ne se rencontrent pas nécessairement dans des aliments

Megenberg, Yconomica, I. 3, 36, éd. cit. de S. Kruger, p. 192-4). De même, l'humaniste italien Bartolomeo Platina, dans son portrait du cuisinier idéal, exige «qu'il connaisse de manière appropriée la puissance et la nature des viandes, poissons et légumes, afin qu'il distingue ce que l'on doit faire rôtir, bouillir ou frire» (cognoscat apposite carnium piscium holerum vim atque naturam ut quid assum quid elixum quid frictum fieri debeat deprehendat: De Honesta Voluptate, I, 11, éd. M. E. Milham, On Right Pleasure and Good Health, Tempe (Az.), 1998 [Medieval and Renaissance Texts and Studies, 168], p. 118).

${ }^{20}$ T. Scully, « Du fait de cuisine par Maistre Chiquart, $1420 »$, Vallesia, 40, 1985, p. 101-231. Id., "Aucune science de l'art de cuysinierie et de cuysine : Chiquart's Du fait de cuisine », Food and Foodways 2-2, 1987, p. 199-214.

${ }^{21}$ Scully, «Du fait de cuisine », art. cit., p. 204.

${ }^{22} \mathrm{~B}$. Laurioux, Le Règne de Taillevent. Livres et pratiques culinaires à la fin du Moyen Âge, Paris, 1997, p. 186.

${ }^{23}$ Un certain Jean de Dudens, notaire : ibid., p. 187.

${ }^{24} \mathrm{R}$. Patni, L'assaisonnement dans la cuisine française entre le XIV et le XVI siècle, thèse de doctorat, ÉHÉSS-Paris, 1989.

${ }^{25}$ L'un des textes médicaux les plus répandus, le Canon d'Avicenne (traduit dans la deuxième moitié du XII ${ }^{\mathrm{e}}$ siècle à Tolède par Gérard de Crémone et entré durant le siècle suivant dans les programmes universitaires), identifie huit saveurs : la saveur douce (dulcedo), la grasse ou 
consommables (mais plutôt dans des médicaments) ou se révèlent insupportables au goût : l'encyclopédiste Barthélemy l'Anglais donne ainsi comme exemple de saveur acerbe le cédrat et de saveur austère le coing pas encore mûr ${ }^{26}$. D'autre part, lorsqu'on prend garde au discours qui s'exprime dans les recettes, on s'aperçoit que le nombre des saveurs y est beaucoup plus limité (le doux, le fort / aigu / épicé, l'acide pour l'essentiel, et quelquefois l'amer) ${ }^{27}$. Il faut donc partir des (rares) recettes culinaires qui mettent en rapport une saveur et des ingrédients ${ }^{28}$.

Même le vocabulaire recèle des pièges. Comme tous ceux qui ont lu des recettes médiévales écrites en moyen français, Terence Scully y a remarqué la fréquence du terme «détremper», qui désigne l'action de mélanger des épices réduites en poudre avec un liquide, tels le verjus, le vinaigre, le vin ou encore le lait et le bouillon $^{29}$. Selon lui, "détremper» a en réalité une signification médicale, liée à la diététique humorale : il s'agirait de compenser la sécheresse et la chaleur excessives des épices afin d'obtenir un mélange équilibré, proche de la nature humaine. Mais l'argumentation de Scully pèche par son ambiguïté : il confond, sans le dire explicitement, temperare et distemperare et, lorsqu'il entreprend d'interpréter le préfixe privatif, la définition de «détremper» comme «to alter the temperament»n'est guère convaincante. Les médecins, pour désigner le tempérament, utilisaient en effet plutôt le terme de "complexion». D'autre part, le vin, le lait et le bouillon n'ont ni les mêmes qualités ni les mêmes effets. En tout état de cause, l'opération qui consistait à équilibrer les qualités humorales par l'adjonction d'un ingrédient donné était rendue par «atremper» et non par « détremper». C'est ce que montrent sans conteste les passages littéraires où les deux termes sont conjointement cités - et ceci dès le XII ${ }^{\mathrm{e}}$ siècle : Cligès ( «Thessala trible sa poison, / Especes i met a foison / Pour adoucir et atanprer / Bien la feit batre et destanprer ${ }^{30}$ ) et Guillaume d'Angleterre :

onctueuse (unctuositas), l'amère (amaritudo), la salée (salsedo), l'acide ou aigre (acetositas), l'austère, c'est-à-dire la resserrée (stipticitas, austeritas), l'acerbe (ponticitas), enfin l'aiguë, la piquante (acuitas). Certains textes leur ajoutent la saveur insipide (insipiditas), ce qui porte à neuf la gamme des saveurs généralement reconnues par les médecins. Cf. C. Burnett, "Sapores sunt octo: The Medieval Latin Terminology for the Eight Flavours ", I cinque sensi. The Five Senses, Florence, 2002 (Micrologus. Natura, scienze e società medievali, X), p. $99-112$.

${ }^{26}$ Barthélemy l'Anglais, Livre des propriétés des choses, traduction française de Jean Corbechon, Paris, BnF, ms. fr 22 532, fol. $325^{\mathrm{r}} \mathrm{a}-327^{\mathrm{r}} \mathrm{a}$.

${ }^{27}$ B. Laurioux, Une histoire culinaire du Moyen Âge, op. cit., chap. 10.

${ }^{28}$ Comme le réceptaire anglo-normand contenu dans le manuscrit London, British Library, Royal 12 C XII (publié par C. B. Hieatt et R. F. Jones, "Two Anglo-Norman Culinary Collections Edited from BL Manuscripts Additional 32085 and Royal 12.C.xii », Speculum, 61, 1986, p. 866-8), ainsi que le manuscrit Firenze, Biblioteca Riccardiana, 1071 (éd. de S. Morpurgo, LVII Ricette d'un Libro di cucina del buon secolo della lingua, Bologne, 1890).

${ }_{29}$ T. Scully, «Deffaire and destremper in Early French Cuisine : The History and Sense of Two Technical Culinary Terms », Petits Propos Culinaires, 38, 1991, p. 14-19.

${ }^{30}$ Chrétien de Troyes, Cligès, v. 3254 sq., cités par A. Tobler-E. Lommatzch, Altfranzösisches Wörterbuch, Berlin, 1915 sq., t. II, col. 1795-97. 
Nature est tex c'onques ne fause. / Tous jors porte avoec li se sause ; / Mais l'une est douce, l'autre amere, [...] / En l'une a girofle et canele / Et cardemome et nois muscades / S'est de jus de pume grenate / Avoec le bausme destempree ; / Et l'autre est si mal atempree / Qu'il n'i a ne cire ne miel ${ }^{31}$

On voit bien qu'ici «détremper» désigne le simple geste par quoi l'on mélange les divers composants d'une préparation, notamment un liquide avec un solide. Si le terme est couramment utilisé dans les recettes médicales, le sens plus neutre de délayer et mouiller un mélange pâteux ou poudreux dans un liquide est également bien attesté ${ }^{32}$ : notamment dès le début du XIV ${ }^{\mathrm{e}}$ siècle pour la peinture ${ }^{33}$. C'est vraisemblablement cette acception large et non savante que les cuisiniers avaient en tête.

Une autre manière de cerner les influences médicales sur les pratiques alimentaires peut être de rechercher dans les textes non culinaires et non médicaux les indices d'un respect des principes diététiques. C'est ce qu'a fait Allen Grieco, en s'appuyant principalement sur les comptes des Seigneurs de Florence au milieu du $\mathrm{XIV}^{\mathrm{e}}$ siècle $^{34}$. Ces comptes ont l'avantage de recenser toute l'alimentation des dirigeants de la commune (qui ne pouvaient être invités en ville ni recevoir de cadeaux $)^{35}$. Or, on y trouve une grande cohérence avec les prescriptions diététiques. Par exemple en ce qui concerne l'utilisation du vin: le vin doux et fort, réputé très chaud, est consommé surtout en été et, d'autre part, les élites semblent préférer le blanc, subtil, au gros rouge laissé aux classes laborieuses ${ }^{36}$. On notera aussi l'usage préférentiel des volatiles pour les classes «oisives", qui ne consomment presque jamais la nourriture grossière (car difficile à digérer) qu'est à cette époque le bœuf (sauf le Mardi Gras et le jour des Innocents, c'est-à-dire lors de rites d'inversion sociale $)^{37}$. Reste un problème d'interprétation, pour les volailles comme pour le vin : les médecins ne se sont-ils pas contentés d'entériner en la matière des goûts qui

${ }^{31}$ Chrétien de Troyes, Guillaume d'Angleterre. Roman du XII siècle, éd. de M. Wilmotte, Paris, 1978 (Classiques français du Moyen Âge, 55), p. 42, v. 1348-1359. La variante çucre pour cire, citée dans Tobler-Lommatzch, Altfranzösisches Wörterbuch, op.cit., t. II, col. 1795-97, est infiniment probable.

${ }^{32}$ Dès le XIII ${ }^{\mathrm{e}}$ siècle, dans le réceptaire médical intitulé Novele cirurgerie, «destremper» a exclusivement le sens de délayer, dissoudre : C. B. Hieatt-R. F. Jones, La Novele Cirurgerie, Londres, 1990 (Anglo-Norman Text Society, 46), passim.

${ }^{33} \mathrm{~F}$. Godefroy, Dictionnaire de l'ancienne langue française et de tous ses dialectes $d u I X^{e}$ au $X V^{e}$ siècle, Paris, 1891-1902, Supplément, t. IX, p. 364a.

${ }^{34}$ A. J. Grieco, Classes sociales, nourritures et imaginaire alimentaire en Italie $\left(X I V^{e}-X V^{e}\right.$ siècles), thèse de $3^{\mathrm{e}}$ cycle, ÉHÉSS-Paris, 1987.

${ }^{35}$ Firenze, Biblioteca Laurenziana, ms. Ashburnham 1216 et Firenze, Archivio di Stato, Camera del Comune, Mensa dei Signori, 13, 15, 17, 26, 68. Cf. l'article pionnier de C. Mazzi, «La Mensa dei Priori di Firenze nel secolo XIV », Archivio Storico Italiano, IV ${ }^{\mathrm{e}}$ série, 20, 1897, p. 336-68.

${ }^{36}$ A. J. Grieco, «Le goût du vin entre doux et amer : essai sur la classification des vins au Moyen Âge ", dans Le Vin des historiens. Actes du $1^{\text {er }}$ Symposium Vin et Histoire, 19, 20 et 21 mai 1989, Suze-la-Rousse, 1990, p. 89-97.

${ }^{37}$ A. J. Grieco, Classes sociales, nourritures et imaginaire alimentaire, chap. I. 
précédaient leur justification théorique? Autrement dit: il y a bien une cohérence, mais il n'est pas assuré que la diététique en soit le principe organisateur.

Allen Grieco a d'autre part attiré l'attention sur les lettres personnelles qui expriment des conceptions diététiques : par exemple celles que le notaire florentin Ser Lapo Mazzei adressait au grand marchand de Prato, Francesco di Marco Datini ${ }^{38}$. Il me semble possible d'ajouter - au hasard des lectures - d'autres textes à ce dossier, tels les récits de voyage. Ainsi, dans les comptes rendus détaillés qu'il a fournis des visites pastorales effectuées par son évêque en Carniole, Styrie et Carinthie ${ }^{39}$, le clerc humaniste Paolo Santonino se montre attentif à la qualité médicale de ce qu'il mange : entre autres exemples pour essayer de faire passer un plat de tripes, il raconte que la troupe s'offrit du bon vin «qui le cuit vite dans l'estomac $»^{40}$.

La transmission des préceptes diététiques hors du milieu médical est une question évidemment fondamentale. Le regretté Jean-Louis Flandrin se l'était posée face à deux phénomènes qu'il convient à mon avis de considérer séparément.

Il y a d'abord, selon les termes même de Flandrin, «la cohérence des idées diététiques avec d'une part le système des représentations du monde et de la société, et d'autre part le système des valeurs gastronomiques $»^{41}$. Or, on vient de le voir, cette cohérence est loin d'être évidente. La position de Jean-Louis Flandrin s'est peu à peu durcie ${ }^{42}$ en une chronologie qu'il faudrait revoir: $\mathrm{du} \mathrm{XIV}^{\mathrm{e}}$ au XVII ${ }^{\mathrm{e}}$ siècle, la gastronomie (définie au sens large des arts de la table) aurait été dépendante de la diététique, avant de s'en affranchir au cours du XVIII ${ }^{\mathrm{e}}$ siècle. Je me demande si, à l'inverse, la diététique n'aurait pas dépendu de la gastronomie dans ses préférences (mais non, bien entendu, dans son principe organisateur). Du reste, Flandrin avait noté le «suivisme » des médecins de l'Époque moderne à l'égard des changements du goût (par exemple ils ont justifié après coup l'habitude, venue d'Espagne et d'Italie, de boire le vin «à la glace», alors que la théorie diététique voulait qu'on le boive à la température du $\left.\operatorname{corps}^{43}\right)$ : pourquoi ce suivisme n'aurait-il pas déjà été de mise au Moyen Âge?

\footnotetext{
${ }^{38}$ Éd. de C. Guasti, Lettere di un notaro a un mercante del secolo $X V$, con altre lettere e documenti, Florence, 1880.

${ }^{39}$ Cf. B. Laurioux, "Les voyageurs et la gastronomie en Europe au $\mathrm{XV}^{\mathrm{e}}$ siècle ", dans Le Désir et le goût. Pour une autre histoire: actes du colloque à la mémoire de Jean-Louis Flandrin, dir. O. Redon, L. Salmann et S. Steinberg, sous presse.

${ }^{40}$ Éd. de G. Vale, Itinerario di Paolo Santonino in Carintia, Stiria e Carniole negli anni 1485-1487 (Codice Vaticano Latino 3795), Cité du Vatican, 1943 (Studi e testi, 103).

${ }^{41}$ J.-L. Flandrin, «Diététique et gastronomie, XIV $-\mathrm{XVIII}^{\mathrm{e}}$ siècles », art. cit., p. 178.

${ }^{42} \mathrm{Cf}$. les deux contributions qu'il a consacrées à (en grande partie) ce sujet dans l'Histoire de l'alimentation co-dirigée avec Massimo Montanari (Paris, 1996): J.-L. Flandrin, "Assaisonnement, cuisine et diététique aux $\mathrm{XIV}^{\mathrm{e}}, \mathrm{XV}^{\mathrm{e}}$ et $\mathrm{XVI}^{\mathrm{e}}$ siècles », p. 491-509 et surtout «De la diététique à la gastronomie, ou la libération de la gourmandise », p. 683-703.

${ }^{43}$ J.-L. Flandrin, «Diététique et gastronomie, XIV ${ }^{\mathrm{e}}-\mathrm{XVIII}^{\mathrm{e}}$ siècles », art. cit., p. 190, corrigeant fortement ce que le même auteur avait écrit antérieurement (J.-L. Flandrin, "Médecine et habitudes alimentaires anciennes », Pratiques et discours alimentaires à la Renaissance. Actes du colloque de Tours de mars 1979, Centre d'études supérieures de la Renaissance, dir. J.-C. Margolin-R. Sauzet, Paris, 1982, p. 86-7).
} 
Plus facile à repérer est la persistance d'habitudes manifestement liées à d'anciennes conceptions diététiques depuis longtemps dépassées. L'exemple du melon est un classique : pourquoi, alors qu'il est fort sucré, le mange-t-on en début de repas, en l'accompagnant (de plus en plus rarement maintenant) de sel et (plus souvent) de jambon salé et / ou en buvant par-dessus un verre de vin fort (porto, muscat, etc.)? La réponse réside dans le statut très particulier qu'occupait le melon dans l'ancienne diététique. Il y était réputé très froid et à ce titre éminemment dangereux. D'autre part, il était soupçonné d'être très putrescible. C'est pourquoi l'on conseillait le consommer en début de repas, afin de donner plus de temps à la digestion (conçue comme une coction) pour l'assimiler. L'usage de vin, de nature chaude, permettait de combattre sa froideur et celui de mets salés la corruption ${ }^{44}$.

Mais comment ces conceptions, somme toute assez complexes, ont-elles pu se transmettre si largement dans la société ? Jean-Louis Flandrin fondait de grands espoirs sur les proverbes. Mais les recherches littéraires ont montré qu'ils ne témoignaient pas plus des «mentalités» populaires que les fabliaux. D'autre part, leur «message gastronomique» est passablement obscur, ambigu voire contradictoire ${ }^{45}$. Si elle a jamais existé, la transmission orale des préceptes diététiques reste pour l'heure impossible à repérer.

Au demeurant, dans les pratiques, l'interventionnisme médical en matière de diète rencontre de puissantes résistances. Jacques Despars s'en étonne dans le passage déjà cité de son commentaire au Canon d'Avicenne ${ }^{46}$. Les médecins médiévaux ont dû composer avec l'obstination des goûts et les ruses du désir alimentaire : c'est ce qu'ont parfaitement compris ceux d'entre eux qui ont écrit des recettes de cuisine que je qualifierais de «médicale $»^{47}$.

Ainsi, Petrus Musandinus, auteur d'un traité sur l'alimentation des malades atteints de fièvres ${ }^{48}$, fournit une variante du lait d'amandes de couleur parfaitement

\footnotetext{
${ }^{44}$ J.-L. Flandrin, Chronique de Platine. Pour une gastronomie historique, Paris, 1992, p. 1239.

${ }^{45} \mathrm{D}$. Rivière, « Le thème alimentaire dans le discours proverbial de la Renaissance française », Pratiques et discours alimentaires à la Renaissance, op. cit., p. 201-17 ; E. Rassart-Eeckhout, "Pratiques alimentaires à la fin du Moyen Âge : l'apport de la langue imagée », La Vie matérielle au Moyen Âge: l'apport des sources littéraires, normatives et de la pratique. Actes $d u$ Colloque international de Louvain-la-Neuve, 3-5 octobre 1996, éd. E. Rassart-Eeckhout et alii, Louvain-la-Neuve, 1997 (Université Catholique de Louvain, Publications de l'Institut d'Études Médiévales, Textes, Études, Congrès, 18), p. 148-77.

${ }^{46}$ «En vérité, en France et dans nos régions, où les habitants sont pour la plupart voraces, il n'est pas habituel d'user d'une diète aussi stricte» (D. Jacquart, La Médecine médiévale dans le cadre parisien, op. cit., p. 433).

${ }^{47}$ B. Laurioux, «La cuisine des médecins à la fin du Moyen Âge », Maladie, Médecines et Sociétés, Approches historiques pour le Présent, Actes du VI colloque d'Histoire au Présent, Paris, 1993, t. II, p. 136-148.

${ }^{48}$ B. Laurioux, "Petrus Musandinus et son traité sur l'alimentation des malades », dans La Scuola Medica Salernitana : gli autori e i testi. Actes du colloque de Salerne, 3-5 novembre 2004, sous presse.
} 
blanche, car «cela plaît davantage aux malades $»^{49}$. Il consacre toute une section de son ouvrage à des plats de viande, formellement interdits aux fiévreux, mais dont ils peuvent rêver dans leur triste régime de salades et d'amandes ${ }^{50}$ : Musandinus conseille d'utiliser alors les cous et les ailes des poules, poulets et faisans - volatiles assez tempérés mais que l'on devra néanmoins "refroidir» avec force jus de grenades, verjus ou vinaigre. La poule est également autorisée, mais au prix d'une surcuisson: bouillie jusqu'à ce que la chair se détache des os, elle sera ensuite réduite en purée dans un mortier ${ }^{51}$. Enfin il prévoit le cas où un malade voudrait goûter la pâte d'une tourte aux amandes dont seul l'intérieur est prescrit dans le traitement ${ }^{52}$ : concession lucide à la gourmandise! Provoquer l'appétit des patients par d'agréables apprêts est une manière de leur faire avaler leurs médicaments / aliments. ${ }^{53}$

\section{Textes médicaux et textes culinaires : le niveau philologique}

Les textes médicaux sont-ils à l'origine des textes culinaires?

C'est la thèse de Ria Jansen-Sieben, qui prend appui sur le fait que la littérature médicale est antérieure à la littérature culinaire pour postuler que la médecine a transformé la cuisine, par exemple en diffusant l'usage des épices (presque toutes de complexion chaude et sèche et favorisant donc la digestion $)^{54}$. Mais il faut prendre garde à ce que l'émergence tardive de la littérature culinaire médiévale est due à des raisons culturelles. Si, durant le haut Moyen Âge, seuls les médecins prenaient la parole, ce n'est pas parce que les cuisiniers n'avaient rien à dire ni que la cuisine n'évoluait pas. Il paraît certain que des recettes consignant les nouvelles préparations (et d'ailleurs aussi les anciennes) ont circulé (oralement). De ce point de vue, il existe un décalage chronologique entre ce que nous donne à lire le livre de cuisine et les pratiques culinaires réelles : les livres de cuisine du premier $\mathrm{XIV}^{\mathrm{e}}$ siècle mettent ainsi en œuvre un répertoire antérieur, que l'on peut retrouver dans les textes littéraires ou les comptes d'approvisionnement ${ }^{55}$.

\footnotetext{
${ }^{49} \mathrm{Si}$ vis quod lac predictum sit magis album, in quo magis delectetur infirmus (éd. de $\mathrm{S}$. De Renzi, Collectio Salernitana, t. V, Naples, 1859 [désormais abrégée en CS], p. 255) : pour ce faire, on utilise de l'eau froide au lieu d'eau chaude.

${ }_{50}$ Contingit in febribus quod paciens febrem acutam debilis est et fastidit predictos cibos et affectat carnem : unde debemus ei satisfacere ut natura confortetur (CS, p. 256-57).

${ }^{51}$ Ibid., p. 257.

${ }^{52}$ Si paciens de pasta comedere voluerit (CS, p. 260). Au lieu de la pâte simple, à base d'eau et de ptisana d'orge, est recommandée une pâte pour artocreas, c'est-à-dire pour pâtés gras de viande (sur les artocreas, cf. A. M. Bautier, "Pain et pâtisserie dans les textes médiévaux latins antérieurs au XIII ${ }^{e}$ siècle ", Manger et Boire au Moyen Âge. Actes du Colloque de Nice, 15-17 octobre 1982, Paris, 1984, t. 1, p. 44).

${ }_{53}$ À la première recette de lait d'amandes, Musandinus recommande d'ajouter un peu de sucre, de pénides ou de sirop, si le malade aime les douceurs (si infirmus delectetur in dulcibus, CS, p. 255). Le souci de faire plaisir aux malades s'étend au service de table : tere eas in pulchro vase, quia, si vas in quo preparatur vel datur infirmo aliquid pulchrum sit, infirmus plus delectatur (Ibid.).

${ }^{54}$ R. Jansen-Sieben, «From food therapy to cookery-book », art. cit.

${ }^{55}$ B. Laurioux, Une histoire culinaire du Moyen Âge, chap. 3.
} 


\section{Il existe bien une cuisine des médecins}

Je ne parle pas ici des nombreuses œuvres médicales qui donnent, depuis l'Antiquité, des listes détaillées d'aliments avec leurs propriété ${ }^{56}$ ni même des régimes de santé qui mentionnent ici ou là des procédés culinaires mais de textes écrits par des médecins et entièrement dévolus à la préparation des aliments.

On a déjà cité la Summula (ou Tractatus) de preparatione ciborum et potuum infirmorum, écrite à l'extrême fin du XII ${ }^{\mathrm{e}}$ siècle par un maître de la fameuse école de Salerne, Petrus Musandinus. L'une de ses premières notices montre que, si l'objectif est de nourrir les malades (notamment ceux atteints de fièvre), la forme est bien celle d'une recette de cuisine. Du reste, référence est faite ici à des plats bien réels de la cuisine «normale», telle la junctata (jonchée) $)^{57}$.

Un autre plat [d'amandes]. Prends (Accipe) du pain frais, bien cuit et propre, auquel tu enlèveras la croûte. Coupe ensuite en trois tranches fines, pose dans un plat, verse par dessus de l'eau chaude en recouvrant d'une autre écuelle. Lorsque les tranches sont bien molles, prends un pilon et écrase bien le tout; ajoute ensuite la purée d'amandes qui est restée lors de la préparation du lait d'amandes, ou bien des amandes supplémentaires, et broie bien le tout. Mets ensuite dans un récipient allongé et étroit, sur des braises à feu doux, en remuant sans arrêt jusqu'à ébullition. Ajoute un peu de sel, de sucre ou de pénides et, si tu le souhaites, du sirop. Garde couvert jusqu'au moment de servir. Ce plat semble presque de la Iunctata ou de la zuccarata. ${ }^{58}$

Un siècle environ après Musandinus, c'est-à-dire vers 1300, un certain Jamboninus de Crémone - un enseignant de médecine de Padoue, Zambonino da Gazzo - traduisait partiellement un texte arabe du $\mathrm{XI}^{\mathrm{e}}$ siècle, joliment intitulé le Chemin de l'exposition des produits employés par l'homme et que son auteur, Abû 'Alî Yahyâ ibn 'Isâ ibn Jazla, avait présenté comme "la description de tous les médicaments, de toutes les boissons [et] de tous les aliments». Le traducteur (en latin) n'a retenu qu'une petite partie de cette grosse œuvre pour un livre qu'il a appelé Liber de ferculis et condimentis, c'est-à-dire «Livre des plats et des condiments ", ou encore (d'après le colophon) Des aliments et des médicaments simples et composés ${ }^{59}$.

${ }^{56}$ Le prototype en est le De facultatibus alimentorum de Galien, qui a lui-même fortement influencé les Diètes d'Isaac, en grande partie organisées par aliments.

${ }^{57}$ Cette sorte de fromage frais est bien attestée dans la cuisine italienne du XIV ${ }^{\mathrm{e}}$ siècle (cf. les recueils édités par F. Zambrini, Il Libro della cucina, Bologne, 1863, p. 78 et O. Guerrini, Frammento di un libro di cucina del sec. XIV, Bologne, 1887, p. 20). La variante de carême, préparée à base de lait d'amandes, est encore plus répandue : éd. d'I. Boström, Anonimo Meridionale. Due Libri di Cucina, Stockholm, 1985, p. 36 et d'E. Faccioli, Arte della cucina. Libri di ricette, t. I, Milan, 1966, p. 171 ; mss de Châlons-sur-Marne, Bibliothèque Municipale, 319, fol. $38^{\mathrm{r}}$, de New York, Pierpont Morgan Library, Bühler 19, fol. $60^{\mathrm{r}}$ et de London, British Library, Addit. 18165, index, $\mathrm{n}^{\circ}$ CII.

${ }^{58} \mathrm{CS}$, p. 255.

${ }^{59}$ Signalé par F. Novati dès 1900 (« Maestro Jambobino da Cremona traduttore dall'arabo fin qui sconosciuto", Archivio storico lombardo, 14, 1900, p. 146-49), le Liber de ferculis et condimentis a été assez fortement mis à contribution, un demi-siècle plus tard, par M. Rodinson (notamment dans «Romania et autres mots en arabe en italien», Romania, 71, 1950, p. 433-49 ; version anglaise remaniée, Petits Propos Culinaires, 34, march 1990, p. 31-44), 
Ce dernier titre traduit bien l'ambiguïté des notices de ce livre dont l'extrait reproduit infra peut donner une idée.

Sumachia. La meilleure est celle que l'on fait à partir de sumac frais et rouge. Elle est froide et sèche. Elle agit contre la faiblesse du ventre qui vient de l'humeur chaude, et resserre le ventre; elle convient contre les crachements de sang et fortifie le sang. Pour qui veut qu'elle ne resserre pas [le ventre] il doit y ajouter des branches de pourpier et des feuilles de cédrat ou des côtes de bettes ou d'épinards. Elle nuit à la poitrine. On lui ôte cet inconvénient [en utilisant en même temps] la Mehelebia.

Elle se prépare ainsi. Coupe de la viande grasse en petits morceaux; mets dans une marmite avec un peu d'huile de sésame, un oignon, un peu de poivre en poudre: mélange assez longtemps. Ensuite coupe de la viande maigre en morceaux larges et fins, mets-les dans la marmite et mélange. Puis arrose peu à peu en plusieurs fois la viande d'eau jusqu'à ce qu'elle soit presque cuite. Prends (accipe) ensuite un peu de clou de girofle et de coriandre et mélange-les avec le reste. Puis mets l'eau dans laquelle le sumac a macéré toute la nuit, bien passée dans un tissu. Enfin ajoute quelques feuilles de menthe et un peu de noix muscade. ${ }^{60}$

On voit clairement que la première partie du texte est consacrée à la caractérisation médicale du plat : la qualité la meilleure (en l'occurrence du sumac, produit assez rare en Occident, plus pharmaceutique que culinaire) ; la place dans le tableau des qualités premières (chaud/froid, sec/humide); les indications thérapeutiques (astringence qui permet de combattre la diarrhée selon toute apparence); les contreindications et les moyens de les atténuer. Ensuite seulement vient la recette qui, là aussi, ressemble beaucoup à une recette qu'on pourrait lire dans un livre de cuisine.

Cette ressemblance doit être appréciée au regard de livres de cuisine contemporains des textes médicaux. Du fait de l'émergence tardive de la littérature culinaire dans l'Occident latin, ce n'est guère possible qu'avec notre troisième texte, l'Opusculum de saporibus, autrement dit l'Opuscule sur les sauces, partie détachée d'un grand régime de santé que le médecin italien Maino de Maineri dédia à son

avant d'être à peu près oublié. Une série de travaux récents a manifesté un regain d'intérêt pour ce traité : E. Carnevale Schianca («Il Liber de ferculis et condimentis, un ricettario di cucina araba nella traduzione di Jambobino da Cremona », Appunti di gastronomia, 35, giugno 2001, p. 5-60) a proposé une traduction italienne du texte latin (ms. Paris, BnF, lat. 9328) et un commentaire fondé sur la transcription du texte arabe qu'avait réalisée le médecin hollandais Pieter de Koning; A. Martellotti (Il Liber de ferculis di Giambonino da Cremona. La gastronomia araba in Occidente nella trattatistica dietetica, Fasano, 2001 [Biblioteca della Ricerca. Cultura straniera, 108]) a utilisé cette même transcription pour commenter l'édition (et traduction) qu'elle a donnée de la version allemande (ms. München, Bayerische Staatsbibliothek, Cgm 415); après quoi E. Carnevale Schianca ("Ancora a proposito di Jambobino e del Liber de ferculis ", Appunti di gastronomia, 38, giugno 2002, p. 11-37) donnait enfin l'édition (à trous) du texte latin, par quoi il aurait fallu à l'évidence commencer ! Il est regrettable que les responsables de ces initiatives dispersées n'aient pas eu les compétences linguistiques leur permettant de traiter l'ensemble d'un dossier qui nécessiterait à l'évidence un travail d'équipe.

${ }^{60}$ Ms. BnF, lat. 9328 , fol. $159^{\mathrm{r}}$ b. 
compatriote évêque d'Arras ${ }^{61}$. Pourquoi avoir consacré toute une partie aux sauces? Parce que, comme l'affirme un autre médecin, «l'autre chose pourquoy on fait saulce a viande est pour donner saveur et appetit de mengier, et tel Regars nez prent point garde a la complexion ne de viande ne de sauce, mais a celle qui le delitte plus. Par ce Regart quelle saulce est bonne et aquelle viande ne doit pas dire physicien $»^{62}$. La complexion est la combinaison des qualités premières (de température et d'humidité) qui peut définir aussi bien un aliment qu'un tempérament. Déterminer celle d'un plat composé est une opération délicate, puisqu'il faut tenir compte non seulement de la complexion des ingrédients (principal, secondaires) qu'on utilise mais aussi des processus culinaires qu'on leur fait subir (le rôtissage est censé assécher, le mijotage humidifier, etc.). De ce point de vue, la recette d'une sauce pour oie rôtie est un modèle de subtilité, qu'on ne soupçonnerait pas à la première lecture.

Le canard, l'oie et les volatiles aquatiques de la même nature doivent, lorsqu'ils sont rôtis, être accompagnés d'une poivrade noire, composée de pain rôti noir, détrempé dans du vinaigre et de foie rôti. Le tout est broyé et mélangé avec le jus du rôti et du verjus, en faisant bouillir jusqu'à épaississement. ${ }^{63}$

On a là une suite de corrections ponctuelles, où la nature de chaque élément vient compenser le déséquilibre créé par le précédent, afin de parvenir, au bout du compte, à une certaine "harmonie humorale». Les produits de base étant en l'occurrence des volatiles aquatiques, une première correction, destinée à atténuer leur excessive humidité, consiste à les assécher en les faisant rôtir. La poivrade noire qui accompagne nos volatiles est également une sauce chaude, à cause de l'épice éponyme qu'une lacune probable du manuscrit a fait disparaître ${ }^{64}$. Le poivre risque-t-

${ }^{61}$ Édité par L. Thorndike («A Mediaeval Sauce-Book», Speculum, 9, 1934, p. 183-98) à partir d'un manuscrit de la Bibliothèque Nationale de Naples (VIII D 35), l'Opusculum de saporibus a été commenté par T. Scully («The Opusculum de Saporibus of Magninus Mediolanensis », Medium Aevum, 57, 1985, p. 178-207), qui a attiré l'attention sur les variantes considérables qu'attestaient différentes versions du Regimen Sanitatis dédié à l'évêque d'Arras, Andrea de Florence. L'ensemble de la tradition (complexe) de ce Regimen Sanitatis a été examiné par M. Nicoud, Aux origines d'une médecine préventive, op. cit., t. 1, p. 206209, 213-18 et surtout 222-28). Une édition critique est maintenant envisageable (et souhaitable).

${ }^{62}$ Paris, BnF, ms. fr. 2017, $\mathrm{f}^{\circ} 191 \mathrm{r}^{\circ}$. Ce manuscrit, essentiellement composé d'œuvres alchimiques, consacre un long développement aux sauces («saulces sont choses qui moult sont de transmutation en la viande et pour ce au Regime de sancté ») : avec les «viandes» froides comme le porc, il recommande par exemple d'utiliser des sauces chaudes telles la sauce verte, la cameline ou la moutarde, selon un principe également présent chez Maino de Maineri : «La première [chose pour laquelle 'sauce est mellee avecq la viande' est] pour atemprer la viande destempree » (c'est-à-dire déséquilibrée, ayant un excès d'humeurs). Je remercie Jean-Patrice Boudet de m'avoir indiqué l'existence de ce manuscrit.

${ }^{63}$ Assaturis autem anatum et anserum et similium degentium in aquis sapor conveniens est piperata nigra composita ex pane assato nigro infuso in aceto et epate assato pistis simul distemperatis cum liquore descendente et agresta, et bulliant omnia simul usque ad spissitudinem (éd. cit. de L. Thorndike, p. 188).

${ }^{64}$ La recette de poivre noir contenue dans le Viandier, qui est d'ailleurs destinée à accompagner l'oie rôtie, intègre en effet du poivre rond (éd. de T. Scully, The Viandier of Taillevent, 
il alors d'introduire une excessive chaleur ? Celle-ci est immédiatement corrigée par $\mathrm{du}$ vinaigre, froid et humide. Le résultat obtenu n'est pas pour autant une absence totale de qualités humorales : l'équilibre recherché par le diététicien sera parfait si une certaine proximité est conservée avec le produit de base-à moins que celui-ci ne soit nocif - et si en même temps on a su éviter l'excès. Résultat propre, d'ailleurs, à satisfaire les goûts des mangeurs aussi bien que les exigences du médecin. L'adjonction du foie et de la graisse tombée dans la lèchefrite ont peut-être des justifications dans la médecine des humeurs (par leur humidité, les foies tempèreraient la sécheresse que présente désormais la sauce); ils concourent surtout, avec l'épaississement de la sauce, à l'équilibre gustatif et sensoriel de l'ensemble. Les sauces pour l'oie, le canard et les oiseaux de rivière rôtis, que nous donnent à lire les livres de cuisine de la même époque, en utilisent au reste les foies et surtout la graisse $^{65}$.

Toutefois, le style et la formulation de ces textes médicaux diffèrent assez sensiblement de ceux des livres de cuisine, comme le montre la confrontation de la recette de galantine de l'Opusculum de saporibus avec celle qui porte le même nom dans un recueil culinaire.

1- La galantine chez le médecin Maino de Maineri

À propos des poissons, il faut savoir que plus ils sont de grosse chair, de digestion difficile, de grande superfluité et de nature liquide et plus ils ont besoin de sauces chaudes et piquantes. Et ce n'est pas seulement vrai pour les poissons mais pour les viandes. D'où il s'ensuit que les poissons bestiaux, et particulièrement le porc de mer rôti ou bouilli, exigent une sauce très chaude et très piquante. On doit appliquer le même principe aux autres poissons selon qu'ils se rapprochent plus ou moins du porc de mer. La saveur qui convient donc au porc de mer est la poivrade noire, bouillie et forte dont la composition se fait de poivre noir, de clou de girofle et de pain trempé dans le vinaigre; et qu'elle soit mélangée (distemperetur) avec le bouillon du poisson. Si l'on veut le conserver pendant plusieurs jours, que l'on fasse une gelée (gelatina) dont la composition est celle-ci : prends de la cannelle, du galanga, du clou de girofle, de la noix muscade - chacun 3 drachmes - la moitié d'un pain grillé - un pain de deux impériaux. Que le pain soit trempé dans du vinaigre et du vin bouilli - un demi quamtenum - et que la gelée soit faite avec de l'eau, le vin de cuisson du vin - le poisson est cuit dans le vin et l'eau. La dite gelée est suffisante pour 10 personnes. [...] Pour de grandes lamproies rôties et des murènes, prends du gingembre blanc, du clou de girofle, du galanga, de la graine de paradis, chacun 3 drachmes, la moitié d'un pain trempé dans le vinaigre. Mélanger avec la graisse du

Ottawa, 1988, p. 87 et 227). La «poivrade noire» accompagne des volatiles aquatiques dans le Recueil de Riom, recette $\mathrm{n}^{\circ} 44$ (éd. de C. Lambert, Le Recueil de Riom et la manière de henter soutillement, Montréal, 1987 [Le moyen français, 20], p. 83). La «Peverata negra» est en revanche très rare dans la cuisine italienne: on n'en trouve une recette que dans la table d'un manuscrit actuellement conservé à Sorengo (Libro A, ${ }^{\circ}$ CXXXVI, éd. d'I. Boström, Anonimo Meridionale, op. cit., p. 3).

${ }^{65}$ La dodine, confectionnée à partir de la graisse des volatiles, accompagne les oies (Mesnagier de Paris, éd. de G. E. Brereton et J. M. Ferrier, Oxford, 1981, p. 176 et 178), les oiseaux de rivière (Ibid., p. 175) et les «Malars de rivière" (Ibid. et Viandier, éd. cit. de Scully, p. 107). 
poisson et du verjus, faites bouillir. Ou bien l'on peut faire [avec la lamproie] la gelée ci-dessus. ${ }^{66}$

2- La galantine dans un livre de cuisine latin du tout début du $\mathrm{XIV}^{\mathrm{e}}$ siècle, le Liber de coquina

Ici il est question (docet) des poissons. Et d'abord de la gelée (galantina) : pour une gelée de poissons, prends du bon vin et un peu de vinaigre écumé. Faire bouillir ensemble et lorsqu'ils bouillent, qu'un gros poisson soit coupé en morceaux en gardant les écailles : fais-le cuire avec. Lorsqu'il sera cuit, ôte-le, et fais bouillir le vin qui reste jusqu'à ce qu'il en demeure seulement le tiers. Puis ajoute du safran et d'autres bonnes épices réduites en poudre avec des feuilles de laurier. Ensuite prends le poisson et enlève-lui les écailles. D'aucuns broient fortement dans un mortier ces écailles avec le vin et passent à l'étamine. Le résidu (colatura) est ajouté à un autre vin, afin qu'il puisse mieux prendre (conglutinari). Et quand cette gelée est refroidie, mets dedans les morceaux de poisson et laisse reposer pendant un jour ou une nuit ou plus encore jusqu'à ce que tout soit pris. Et ainsi on peut longtemps conserver le poisson. ${ }^{67}$

On reconnaît entre ces deux versions des points communs, qui ne sont pas sans importance : la recette s'ouvre par l'action de rassembler les ingrédients exprimée dans accipe ou recipe; l'ordre des procédures culinaires est en gros semblable. Mais ce sont les divergences qui l'emportent. Pour le médecin, l'espèce de poisson à cuisiner doit faire l'objet d'une longue mise au point car sa «complexion» détermine les opérations de cuisson et d'assaisonnement qu'il faut lui appliquer - en l'occurrence le porc de mer (c'est-à-dire le marsouin) ${ }^{68}$ peut être traité comme une viande - alors que le cuisinier laisse à l'appréciation de ses confrères le choix ultime de l'ingrédient principal : un bon professionnel doit savoir s'adapter aux conditions du lieu et du moment ainsi qu'aux goûts spécifiques du maître. De même, autant la connaissance de la proportion précise dans laquelle on combine les ingrédients est vitale aux yeux du médecin, autant le cuisinier s'attardera sur les techniques de préparation et sur le résultat à obtenir - le problème-clé de cette recette étant la prise en gelée, par l'effet d'éléments riches en gélatine - telles les parures de poisson. L'insistance sur l'utilisation de cette gelée comme moyen de conservation du poisson n'est pas non plus anodine : les premiers réceptaires de cuisine furent sans doute composés pour les besoins des maîtres d'hôtel des grandes maisons princières ${ }^{69}$.

Malgré leur esprit différent, ces recettes traitent bien de la même préparation et ont sans doute, en dernière analyse, la même origine. Est-ce le texte culinaire qui a puisé dans le texte médical ou l'inverse ? Je pencherais plutôt pour la seconde hypothèse. Les cuisiniers ne savent pas lire, alors que nombreux furent les médecins

${ }^{66}$ Éd. de L. Thorndike, «A Mediaeval Sauce-Book», art. cit., p. 188.

${ }^{67}$ Éd. de M. Mulon, «Deux traités inédits d'art culinaire médiéval», Les problèmes de l'alimentation, Actes du 93 ${ }^{e}$ Congrès national des Sociétés savantes, Tours, 1968, I, Bulletin Philologique et Historique (jusqu'à 1610) du Comité des Travaux Historiques et Scientifiques, 1968, p. 413.

${ }^{68}$ Cf. l'article récent de J.-C. Fichou et O. Levasseur, «Pêcheurs contre "Marsouins" et "Belougas", XVI-XX ${ }^{\mathrm{e}}$ siècle », Food \& History, 2-2, 2004, p. 53-8.

${ }^{69}$ B. Laurioux, Le Règne de Taillevent, op. cit., p. 227-28. 
à posséder des livres de cuisine. Ils leur servaient peut-être à préparer leurs cours et à comprendre les termes employés par les autorités sur lesquelles ces cours étaient appuyés: Erhard Knab, enseignant à la faculté de médecine de Heidelberg et commentateur des Diètes d'Isaac possédait ainsi le Liber de coquina ${ }^{70}$ et son confrère Martin Rentz, lecteur du De honesta voluptate, avait procédé au même exercice universitaire ${ }^{71}$. Il n'est pas exclu que les livres de cuisine aient également servi dans la pratique : un dicton, que le Moyen Âge attribue à Avicenne, n'affirme-t-il pas : "Ce qui a bon goût nourrit le mieux, car la nourriture que l'on mange avec plaisir sera bien reçue dans l'estomac [tandis que] la nourriture mangée sans plaisir ne se digère pas bien »? On imagine assez mal aujourd'hui que son médecin puisse avoir le dernier Ducasse ou le dernier Robuchon dans sa bibliothèque de travail.

De leur côté, les livres de cuisine ont pu intégrer des fragments de discours médicaux. Dans un recueil à visée encyclopédique comme le Tractatus de modo praeparandi et condiendi omnia cibaria, la diététique structure le traité: une partie est consacrée aux nourritures grossières tout juste bonnes pour le bas peuple, une aux mets délicats pour oisifs, une autre aux préparations pour malades ${ }^{72}$; d'autre part, le discours médical y irrigue un nombre non négligeable de recettes ${ }^{73}$. Mais pour d'autres livres, l'apport se limite à quelques recettes destinées aux malades, d'ailleurs très simplifiées si on les compare à celles de Musandinus : malades, mais de quoi dirait un médecin ? En fait ce sont des préparations pour convalescents, à base de bouillon de poule et de sucre ${ }^{74}-$ ce que semblent confirmer les comptes d'hôpitaux.

On aimerait savoir comment ces passages conceptuels et textuels ont pu s'effectuer. Faut-il invoquer un hypothétique contenu médical dans les études effectuées à la faculté des arts - qui concernaient à la fin du Moyen Âge un nombre croissant d'individus ? Ou bien la large diffusion de manuels élémentaires de santé et d'hygiène? On n'a pas fini de chercher ni d'explorer les ponts entre médecine et cuisine médiévales.

Bruno Laurioux

Université de Versailles-Saint-Quentin-en-Yvelines

\footnotetext{
${ }^{70}$ Ms. Città del Vaticano, Biblioteca Apostolica Vaticana, Pal. lat. 1140 et 1768. Cf. C. JeudyL. Schuba, «Erhard Knab und die Heidelberger Universität im Spiegel von Handschriften und Akteneinträgen », Quellen und Forschungen aus Italianischen Archiven und Bibliotheken, 61, 1981, p. 60-108.

${ }^{71}$ Ms. Città del Vaticano, Biblioteca Apostolica Vaticana, Pal. lat. 1141 et 1303 . Cf. M. Nicoud, Aux origines d'une médecine préventive, op. cit., t. 3, p. 627 et 762 ; M. E. Milham, On Right Pleasure and Good Health, op. cit., p. 66.

${ }^{72}$ Notandum est quod quedam cibaria conueniunt et apta sunt nobilibus et diuitibus in quiete existentibus, et ut sunt perdices et fasiani...; quedam uero apta sunt hominibus robustis et in labore existentibus; ut carnes bouine et arietine...; quedam uero infirmis et debilibus uelud gruelus de riso uel auena uel ordeo factus... (éd. de M. Mulon, «Deux traités inédits d'art culinaire médiéval», art. cit., p. 384).

${ }^{73} \mathrm{Cf}$. par exemple à la fin d'une recette de porcelet rôti : Quia cibus est fleuaticus et corruptibilis in stomaco : unde indiget bono condimento (ibid., p. 388).

${ }^{74}$ Voir notamment la brève série des plats pour malades du Viandier qui est, dans les manuscrits les plus anciens, la seule à comporter du sucre.
} 